\title{
Spécificités hydrogéologiques des hautes vallées alpines: Exemple de la Haute-Sarine (Suisse)
}

\author{
Julien Vaudan ${ }^{1}$, Aurèle Parriaux $^{1}$, Laurent Tacher $^{1} \&$ Gianni Della Valle ${ }^{2}$
}

Key words: Quaternary alpine aquifers, typology, resource management

\section{RESUME}

\begin{abstract}
La vallée alpine de la Haute-Sarine est à plus d'un titre représentative des aquifères alluviaux de montagne, de par son environnement comme ses caractéristiques hydrogéologiques. Les investigations et les analyses réalisées dans le cadre de cette étude nous ont permis d'améliorer les connaissances sur le remplissage quaternaire meuble de cette vallée, ainsi que de mettre en évidence l'alimentation préférentielle de la nappe non seulement par l'infiltration de la rivière et des précipitations mais aussi par les apports souterrains des karsts évaporitiques. Nous avons également pu constater la richesse des ressources en eaux souterraines de la Haute-Sarine, tant au point de vue qualitatif qu'au point de vue quantitatif. La connaissance de ces caractéristiques typiques des nappes alluviales de haute altitude a par la suite permis l'élaboration d'un concept de gestion et de protection des eaux souterraines adéquat qui s'inscrit dans une politique de développement durable des ressources dans le contexte alpin.
\end{abstract}

\begin{abstract}
The alpine valley of the Haute-Sarine is on several accounts representative of the alluvial aquifers of mountain by its very environment and its hydrogeologic characteristics. The investigations and the analysis carried out within the framework of this study enabled us to improve knowledge on the Quaternary filling of the valley and to highlight the preferential supplies of the aquifer by infiltration of the river and precipitations and also by the underground contributions of evaporitic karsts. We also could note the opulence of groundwater resources of the Haute-Sarine both from the qualitative and the quantitative points of view. The knowledge of these typical characteristics of alluvial aquifers of high altitude allowed finally the development of a concept of management and protection of groundwater resources that falls under a sustainable development policy of the groundwater storage in the alpine areas.
\end{abstract}

\section{Introduction}

Les vallées de haute altitude représentent la majorité des vallées alpines latérales. Actuellement, les ressources en eaux souterraines y sont essentiellement exploitées de manière gravitaire par le biais de captages de sources situées à l'amont des localités. Les besoins en eau actuels ne nécessitant souvent pas un recours à d'autres ressources, la plupart des collectivités publiques ne font donc pas usage des ressources contenues dans les dépôts quaternaires qui comblent les vallées. Il est néanmoins nécessaire d'appréhender avec plus de précision ces réserves d'eau perchées dans les vallées de haute altitude, et ce notamment dans un cadre de développement durable des ressources naturelles. Non seulement on peut envisager l'exploitation future de ces réserves, mais le développement intense des activités touristiques dans ces vallées et l'urbanisation relativement importante qu'elles induisent (infrastructures routières, tunnels, enneigement artificiel, etc...) constituent des atteintes potentielles aux ressources en eau souterraine qu'il s'agit d'identifier ex ante afin de pouvoir, le cas échéant, réagir avec les moyens appropriés.

Le but de cette étude, outre le fait d'étudier les caractéristiques hydrogéologiques de la Haute-Sarine (considérée comme représentative de ces vallées de part sa situation géographique et géologique) est de déterminer les spécificités des aquifères alluviaux de montagne afin d'élaborer un concept global de gestion et de protection de leurs ressources qui permettrait une éventuelle exploitation dans une optique de développement durable comme cela a été fait par l'Office de l'Economie Hydraulique et Energétique (OEHE) du canton de Berne. Il est ainsi possible de répondre aux exigences imposées par la loi suisse qui veut que les bases scientifiques nécessaires soient produites par les autorités cantonales dans le but d'organiser la gestion des eaux souterraines (art. 58 de la Loi sur les Eaux).

\footnotetext{
${ }^{1}$ Laboratoire de géologie de l'ingénieur et de l'environnement (GEOLEP), Ecole polytechnique fédérale de Lausanne, CH-1015 Lausanne, Suisse. E-mail: aurele.parriaux@epfl.ch

${ }^{2}$ Neuenenstrasse 4, CH-3066 Stettlen, Switzerland. E-mail: della.valle@gmx.ch
} 


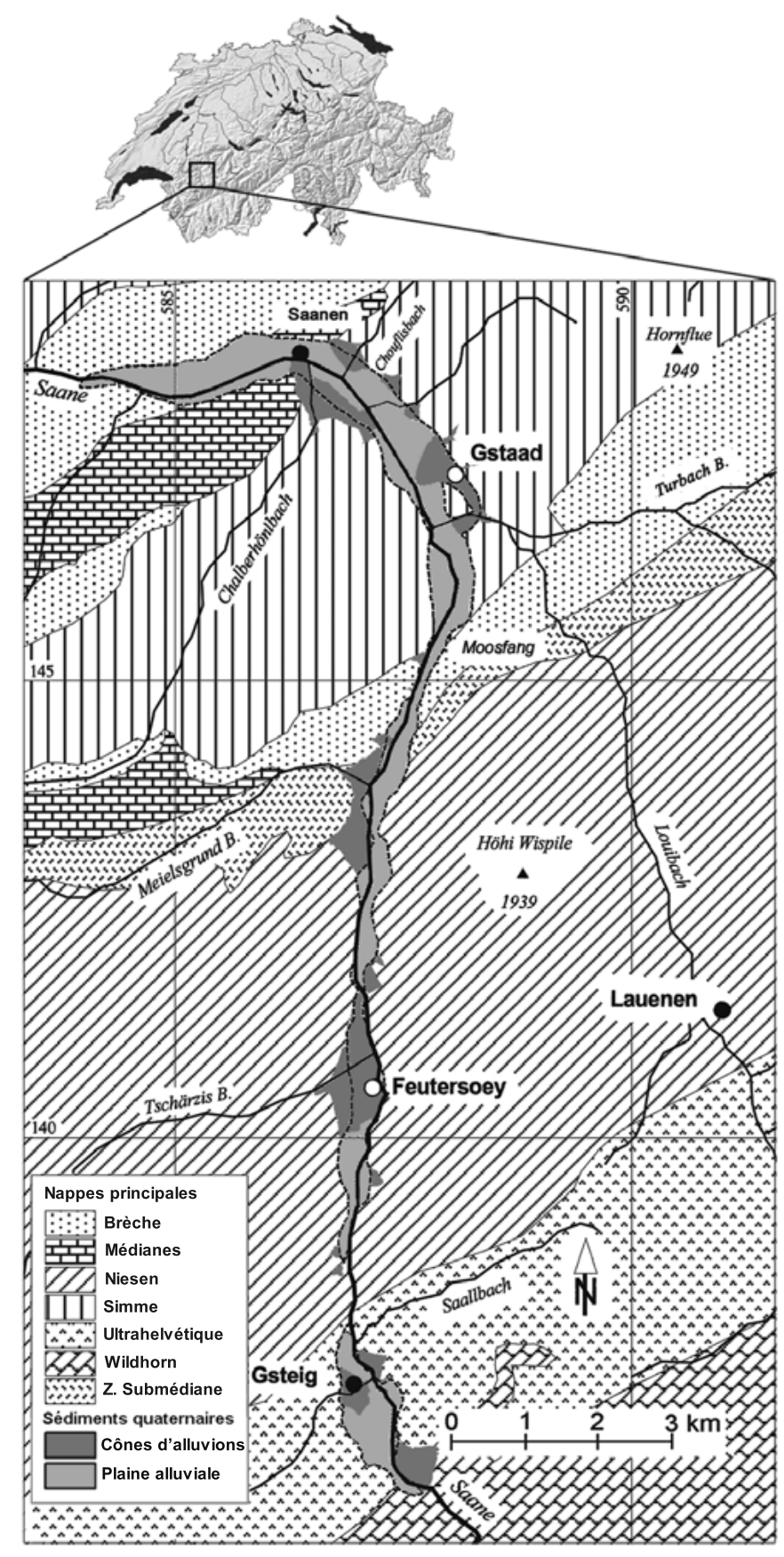

Fig. 1. Contexte géologique de la région de la Haute-Sarine.

\section{Géologie générale et quaternaire}

La vallée de la Haute-Sarine se situe dans les Préalpes Romandes, au nord de l'édifice alpin suisse. Elle est constituée de roches calcaires et évaporitiques appartenant aux nappes préalpines (Fig. 1) et est marquée par un chevauchement vers le Nord d'azimut E-O ainsi que par le grand décrochement N-S que suit la vallée (Flück, 1973).

Le remplissage quaternaire de la vallée (Fig. 2 et Fig. 3) est

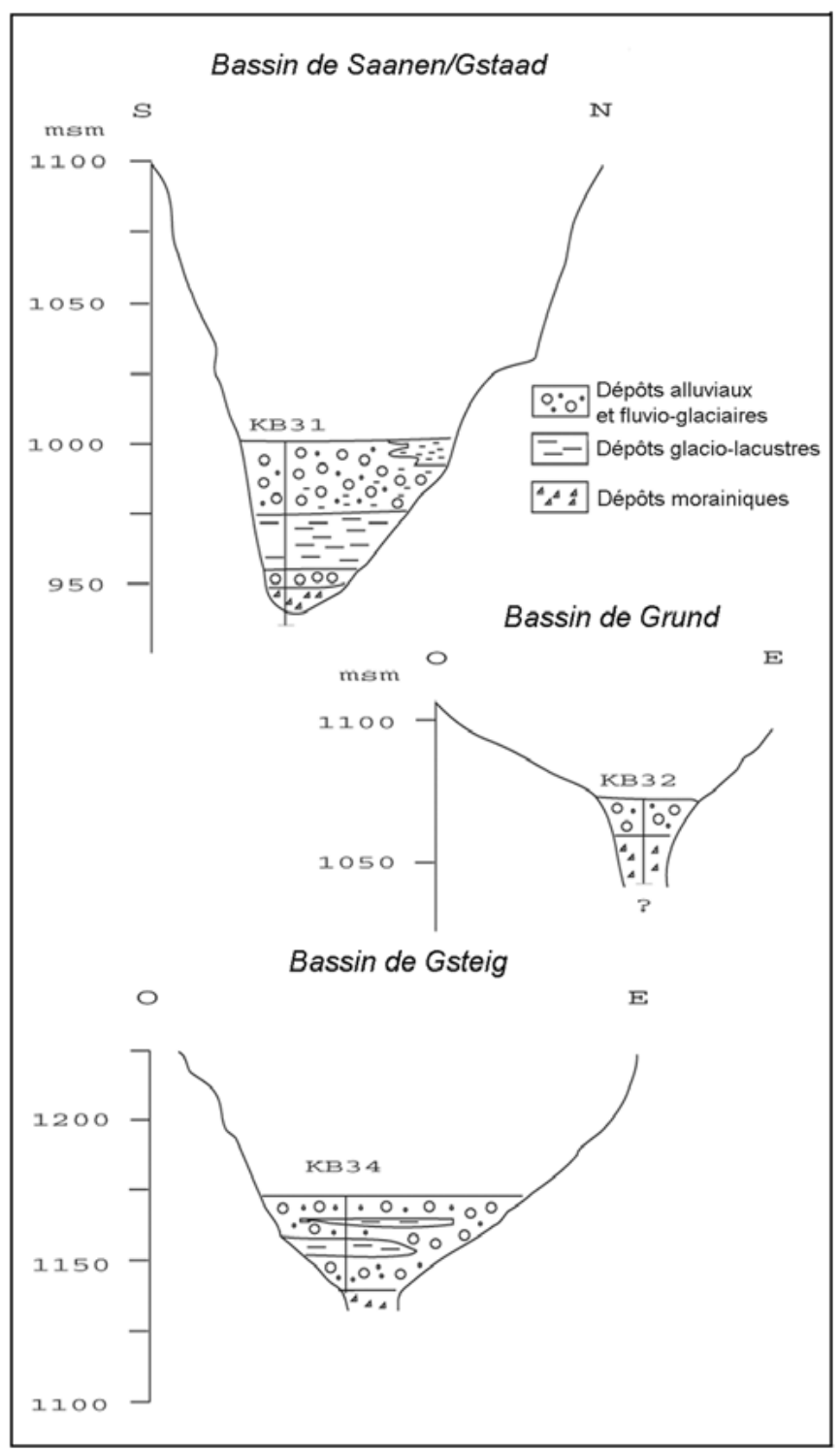

Fig. 2. Profils schématiques des trois segments de la vallée de la Haute-Sarine.

caractérisé par une séquence tardi- et post-glaciaire typiquement alpine (Maisch, 1982). L'épaisseur et la nature des dépôts ainsi que la profondeur de la vallée (Fig. 3) ont pu être déterminées par des approches de géophysique (sismique réfraction et réflexion, investigations géoélectriques; Fig. 4 et Fig. 5; Vaudan, 2000) et par la réalisation de quatre forages complémentaires à ceux déjà existants (KB31, KB32, KB33 et KB 34; Fig. 6; Vaudan, 2000). Dans le bassin de Saanen, la vallée est comblée par des sédiments glaciolacustres surmontant une moraine de fond présentant des chenaux sous-glaciaires graveleux (dépôts tardi-glaciaires). Dans le reste de la vallée, on trouve des dépôts sablo-graveleux de type sandur surmontés de couches de graviers relativement propres à blocs typiquement alluviaux (dépôts tardi- et post-glaciaires). Le retrait des glaciers a également laissé d'importantes formations mo- 


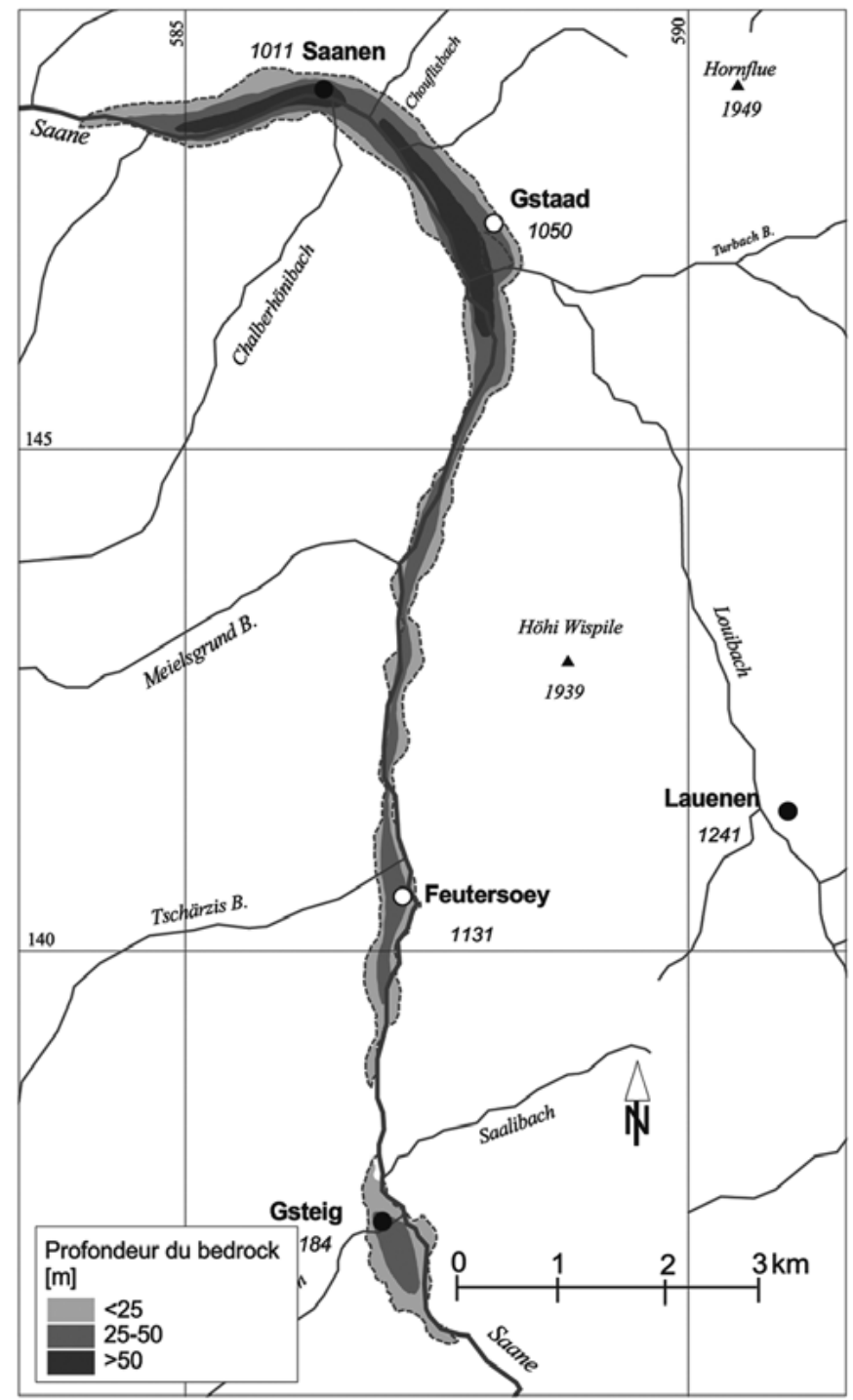

Fig. 3. Carte de la profondeur du bedrock déterminée par les méthodes d'investigation géophysique et par forages.

rainiques qui tapissent le fond et les bords de la vallée. En partie remaniés, ces dépôts se mélangent aux graviers dans les couches les plus profondes.

L'aquifère principal siège dans les dix à vingt-cinq premiers mètres de sédiments et se décompose en trois segments: le segment de Gsteig au sud, fermé à son extrémité nord par un important glissement de terrain, le segment de Feutersoey, fermé à son extrémité nord par l'imposant cône d'alluvions du Tschärzisbach et le segment continu de Grund-Gstaad-Saanen fermé par le verrou rocheux imperméable du Vanel.

\section{Types d'alimentation de la nappe}

L'étude réalisée a permis d'identifier deux principaux types d'alimentation de la nappe (résumés dans la figure 7) que sont

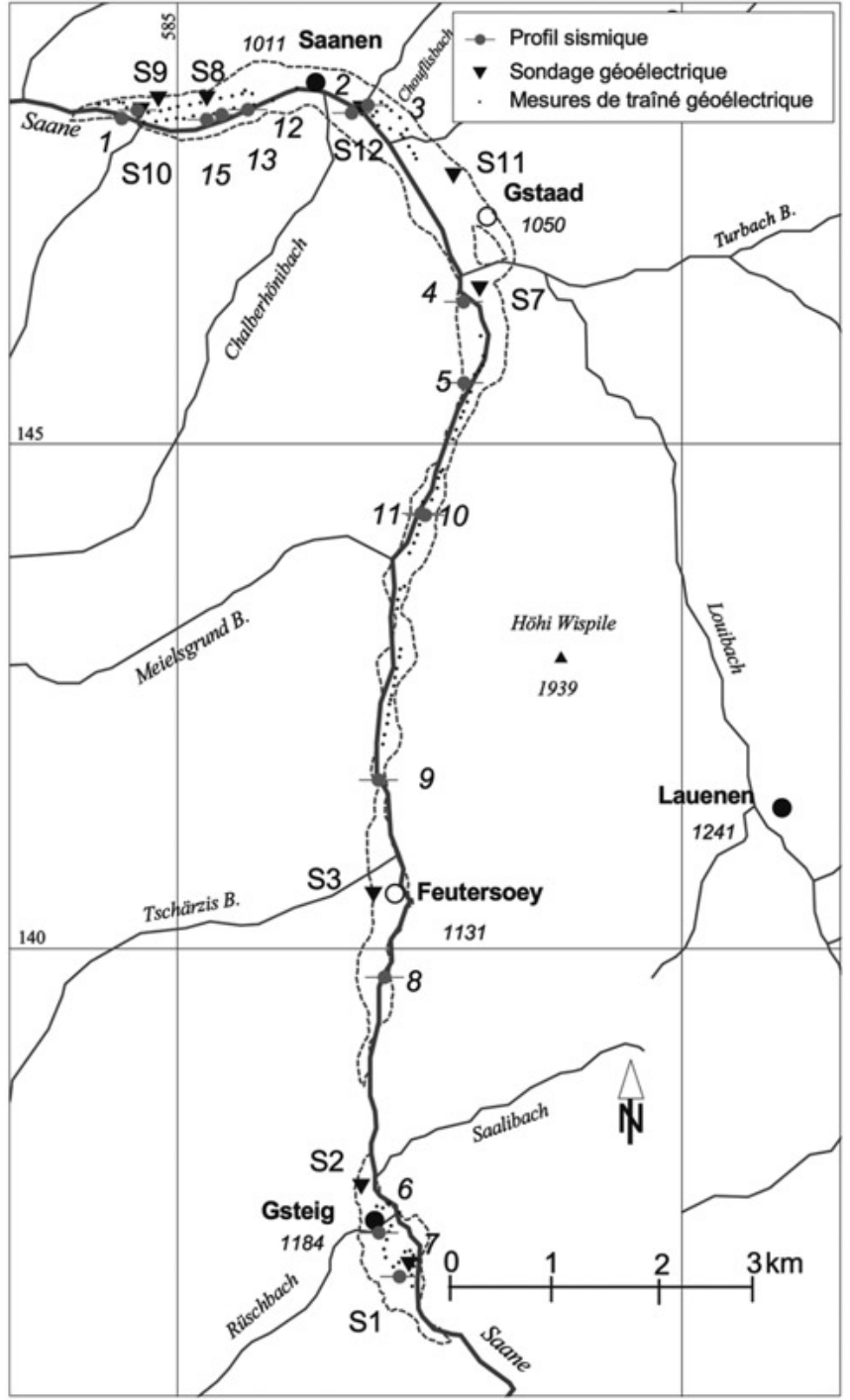

Fig. 4. Carte de situation des sondages et des traînés géoélectriques ainsi que des emplacements des profils sismiques. Les données de terrain sont disponibles au GEOLEP.

l'infiltration directe des précipitations dans la zone saturée de l'aquifère et les alimentations indirectes de types A (infiltration et exfiltration au travers du lit et des rives de la Sarine) et $\mathrm{B}$ (alimentation par les exutoires des réseaux karstiques).

\subsection{Alimentation directe}

L'infiltration directe des précipitations dans l'aquifère se fait de manière extrêmement rapide, comme le montrent les montées abruptes du niveau de la nappe lors d'évènements pluvieux (Fig. 8).

Si l'on considère une évapotranspiration annuelle moyenne de 450 mm (Menzel et al., 1989; Primault, 1972), on obtient des précipitations efficaces de l'ordre de $1200 \mathrm{~mm}$ par an (cf. tableau 1). Le tableau 2 présente les résultats des calculs d'infil- 


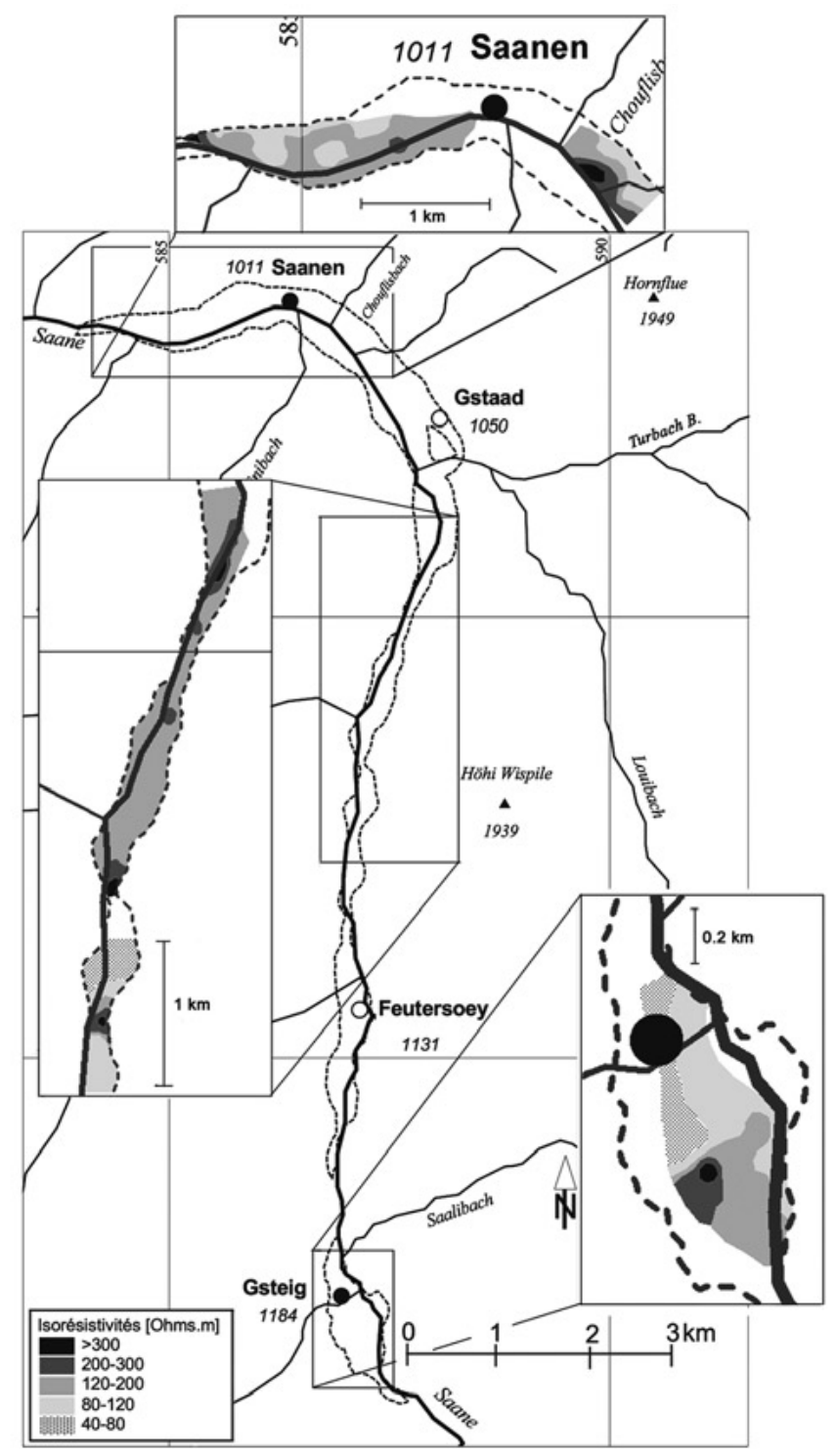

Fig. 5. Carte des résistivités électriques apparentes (traînés électriques TEL de type Schlumberger $\mathrm{AB}=100 \mathrm{~m}$ ); les zones étudiées ont été agrandies.

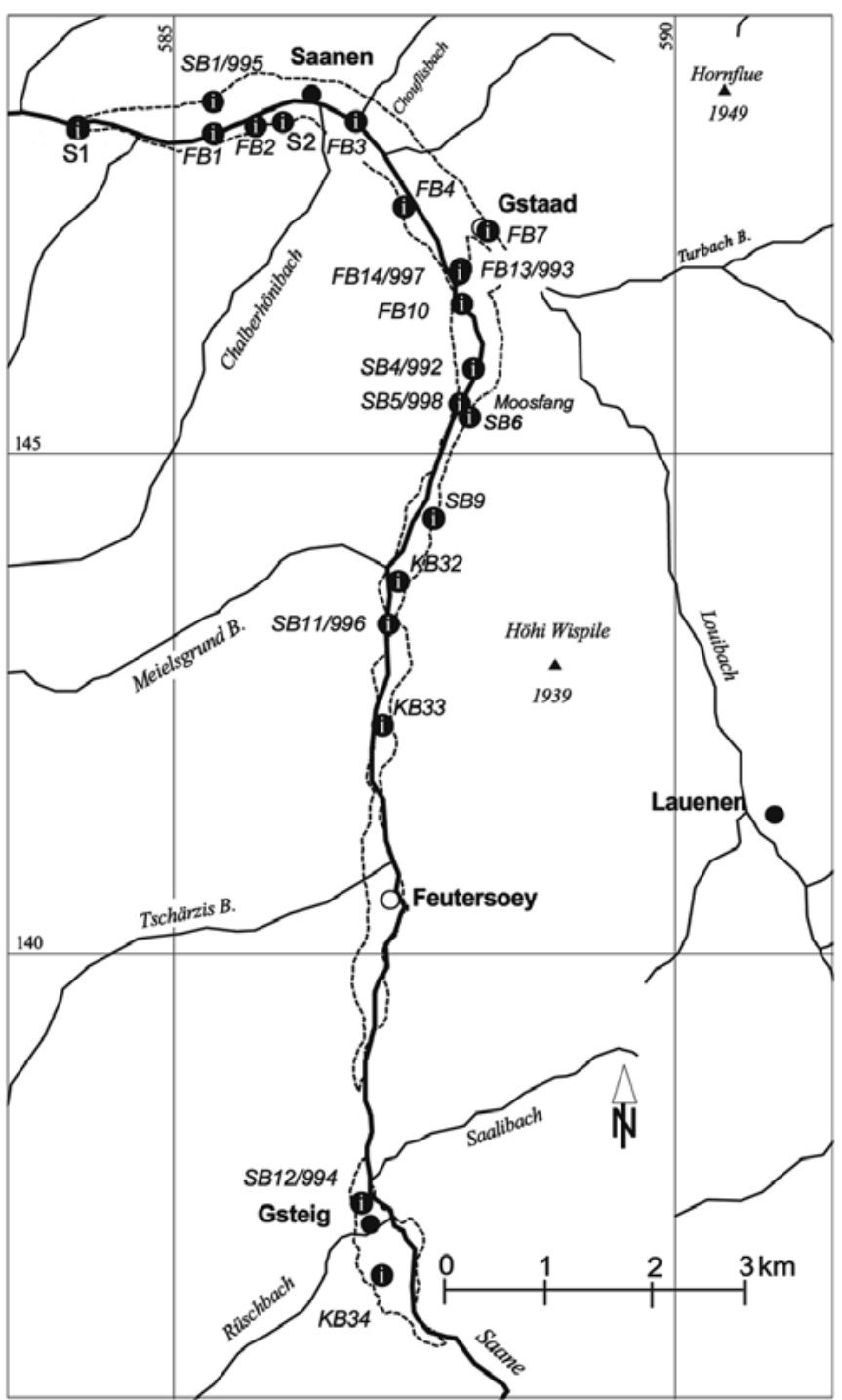

Fig. 6. Carte de situation des sondages mécaniques effectués dans la plaine alluviale de la Haute-Sarine entre 1950 et 1999.
Tab. 1. Précipitations mesurées et précipitations efficaces évaluées dans la zone d'étude.

\begin{tabular}{|lll|}
\hline Années & $\begin{array}{l}\text { Précipitations moyennes } \\
\text { annuelles [mm] } \\
\text { Gsteig } \\
\text { (SMA5560) }\end{array}$ & $\begin{array}{l}\text { Gstaad } \\
\text { (SMA5588) }\end{array}$ \\
\hline 1996 & 1600.7 & 1302.6 \\
1997 & 1648.5 & 1550.8 \\
1998 & 1593.7 & 1373.2 \\
1999 & 2035.0 & 1859.0 \\
Précipitations moyennes [mm] & 1719.5 & 1521.4 \\
Précipitations efficaces & & \\
moyennes [mm] & 1269.5 & 1071.4 \\
\hline
\end{tabular}

tration sur les différentes parties de l'aquifère; on y constate que l'alimentation directe sur l'ensemble de celui-ci s'élève à $5.5 \mathrm{mio}^{3}$ par an, soit l'équivalent d'un débit fictif constant de $173 \mathrm{l} / \mathrm{s}$.

\subsection{Alimentation indirecte}

\subsubsection{Quantification par calcul des bilans des écoulements} souterrains

Pour élaborer une synthèse régionale des ressources en eaux souterraines de la Haute-Sarine, nous avons adopté une mé- 


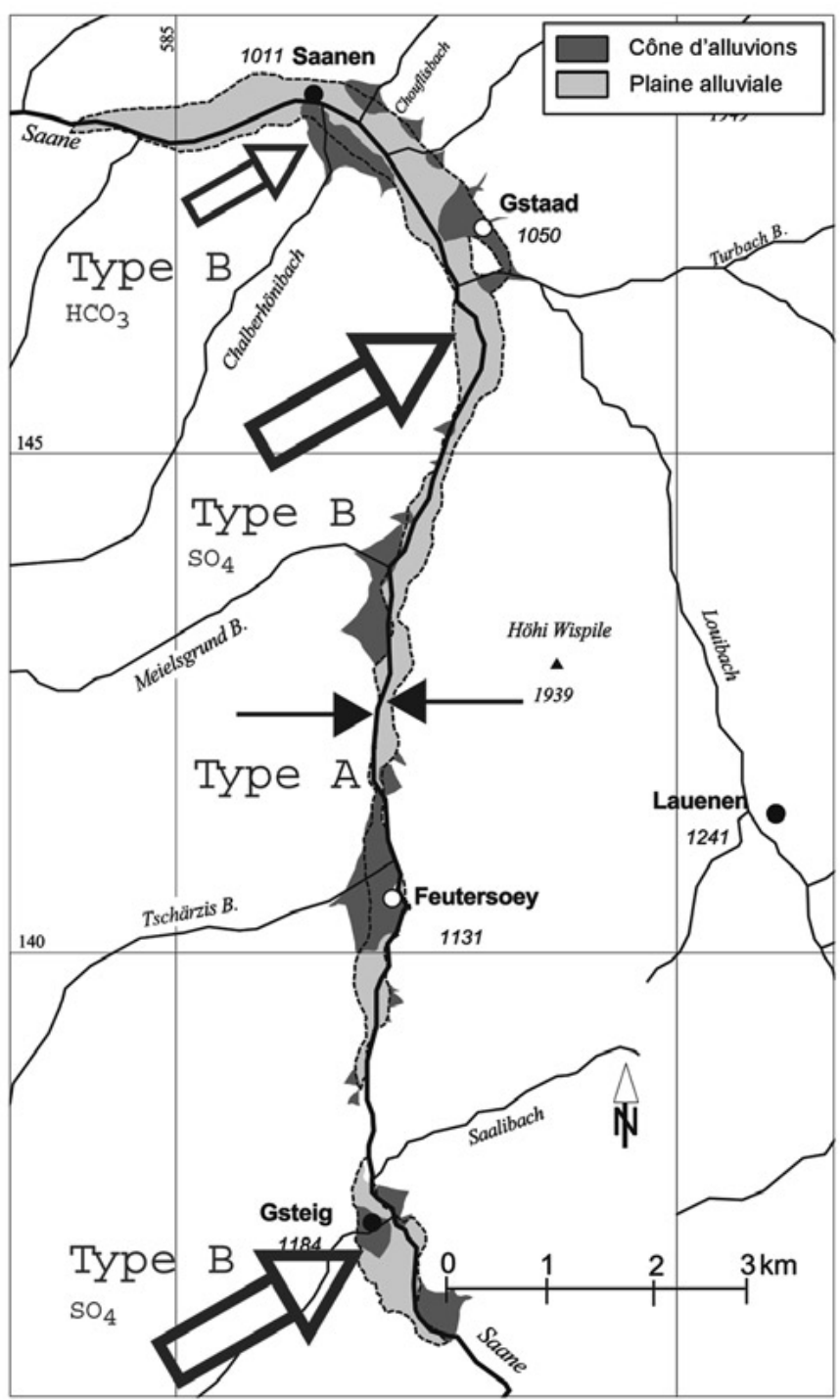

Fig. 7. Représentation schématique des types d'alimentation indirecte de la nappe.

Tab. 2. Calcul de l'alimentation directe annuelle de la nappe (les bassins sont représentés sur la figure 3).

\begin{tabular}{|llll|}
\hline Bassin & $\begin{array}{l}\text { Surface de la } \\
\text { plaine alluviale } \\
{\left[\mathbf{m}^{\mathbf{2}}\right]}\end{array}$ & $\begin{array}{l}\text { Précipitations } \\
\text { efficaces } \\
{[\mathbf{m m} / \mathbf{a}]}\end{array}$ & $\begin{array}{l}\text { Recharge } \\
{\left[\mathbf{M i o .} \mathbf{~ m}^{\mathbf{3}} / \mathbf{a}\right]}\end{array}$ \\
\hline Gsteig & $666^{\prime} 500$ & 1300 & 0.9 \\
Grund & $750^{\prime} 670$ & 1100 & 0.8 \\
Saanen & $3^{\prime} 418^{\prime} 140$ & 1100 & 3.8 \\
Total & $4^{\prime} 835^{\prime} 310$ & & 5.5 \\
\hline
\end{tabular}

thode utilisée de manière standard dans les rapports de l'Office de l'Economie Hydraulique et Energétique depuis plusieurs décennies et qui consiste à comparer les bilans de flux au travers de profils choisis (Fig. 9). Le calcul des bilans s'effectue

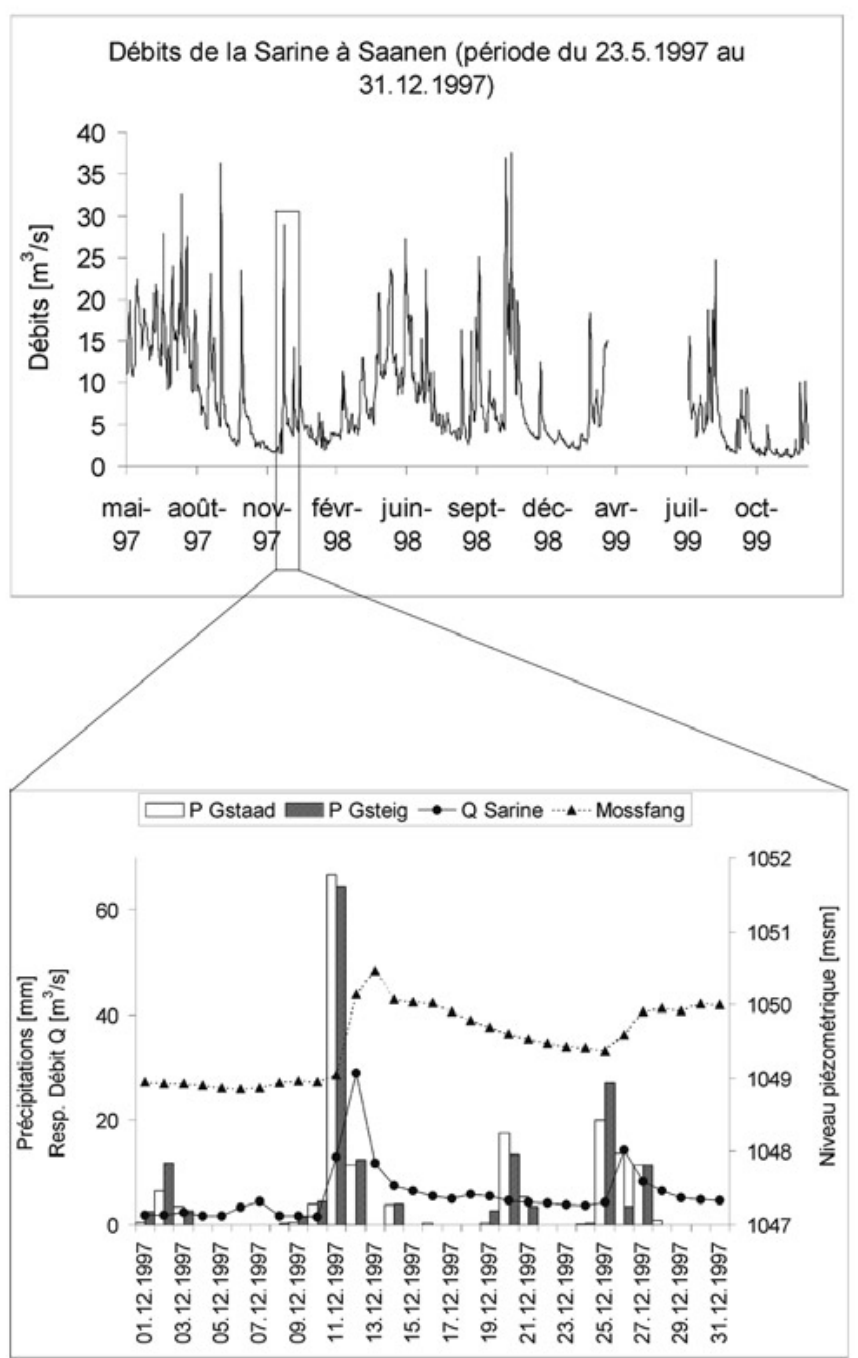

Fig. 8. Exemple de la simultanéité quasi parfaite entre événement pluvieux, crue de la Sarine et crue dans la nappe alluviale pour l'événement du 10.12.1997 au 13.12.1997.

sur la base de la formule de Darcy (Blau et al., 1984; Fürst et al., 1990; OEHE, 1983):

$$
Q=i K F,
$$

où i est le gradient de la nappe, $\mathrm{K}$ la perméabilité en $\mathrm{m} / \mathrm{s}$ et $\mathrm{F}$ la section saturée en $\mathrm{m}^{2}$.

Les données utilisées sont présentées dans le tableau 3, les valeurs de perméabilité ayant été mesurées lors d'essais de pompage et de tests au moulinet de forage (Fig. 10).

Les incertitudes sur les valeurs des différents paramètres utilisés pouvant être importantes étant donné le caractère fortement hétérogène et anisotrope du milieu, nous avons effectué des tests de sensibilité de ces paramètres (Fig. 11) et avons retenu les valeurs moyennes pour effectuer les calculs dans le cas de base. 


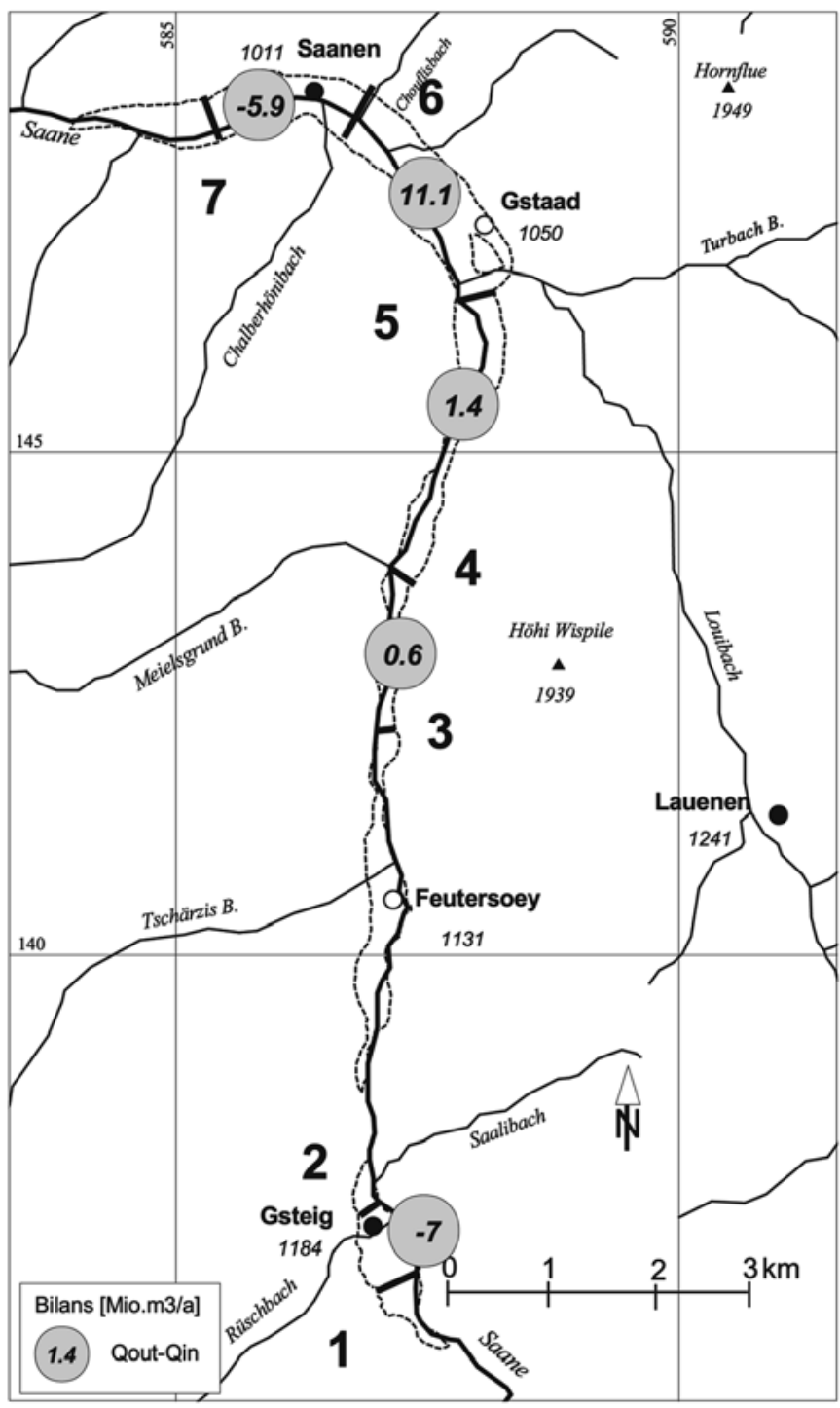

Fig. 9. Situation et numéros des profils de bilan de flux de la nappe. Le calcul du bilan est également représenté entre le profil amont et le profil aval (QoutQin) pour le cas de base du tableau 3.

Ces approches globales ont permis d'obtenir des ordres de grandeur de l'infiltration et de l'exfiltration: en soustrayant les apports souterrains par les versants karstiques évalués à 100 1/s (Mandia, 1991; OEHE, 1999a), on obtient une valeur moyenne proche de 40 1/s pour l'infiltration; l'exfiltration s'élève quand à elle à environ 200 1/s. Ces ordres de grandeur corroborent les différentes mesures effectuées de manière directe ou indirecte lors des nombreuses prospections hydrogéologiques réalisées dans le cadre des rapports sur l'aménagement des eaux du canton de Berne ou lors d'autres prospections d'eau souterraine en milieu alpin (Nicoud et Maillet-Guy, 1990; OEHE, 1983; OEHE, 1989; OEHE, 1994b; OEHE, 1999).

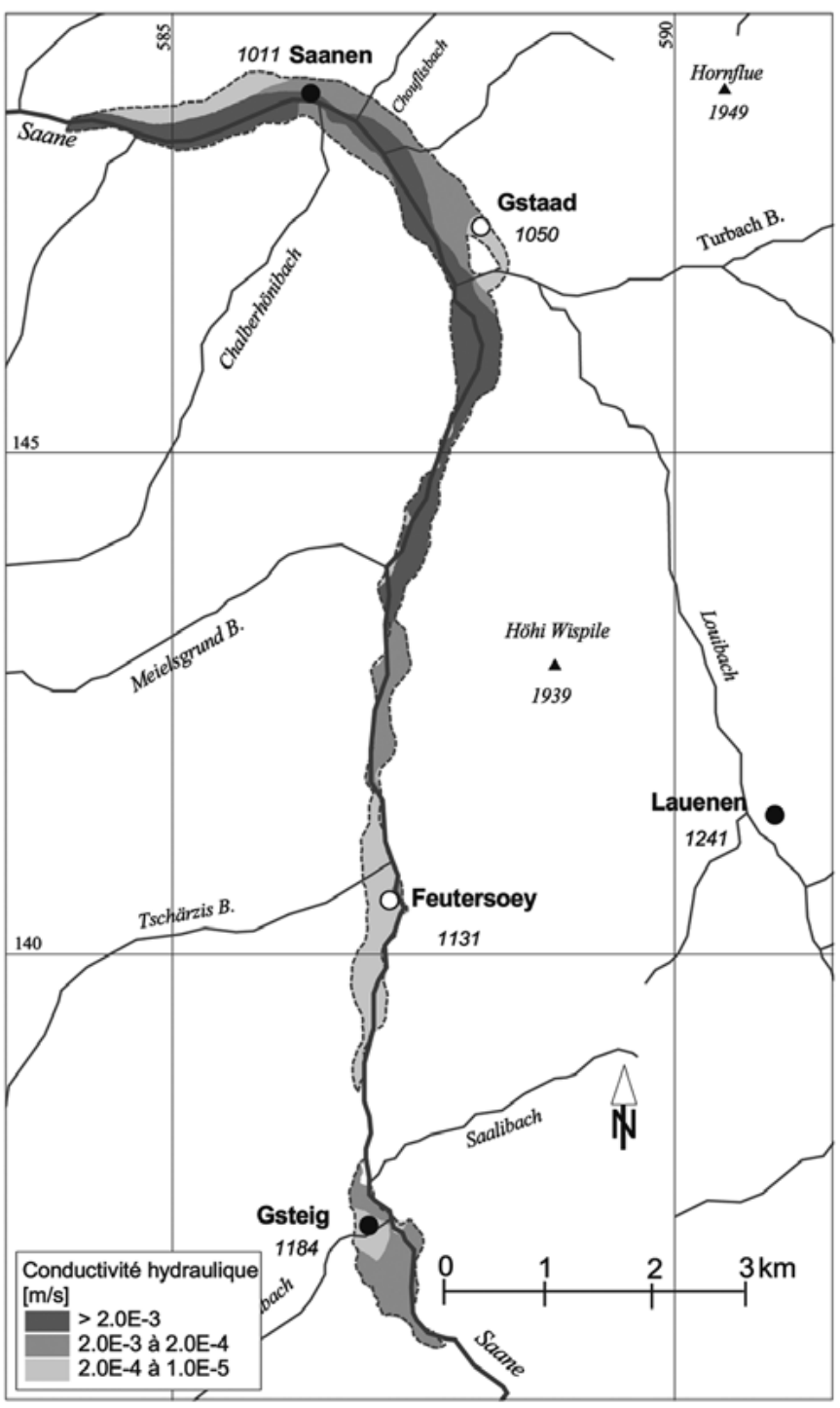

Fig. 10. Carte des conductivités hydrauliques (compilation des archives et de nos essais sur KB31, KB32, KB33 et KB34).

\subsubsection{Quantification par modélisation des écoulements}

Nous avons utilisé le logiciel FEFLOW (Diersch, 1998) afin de tester l'existence d'apports souterrains et en particuliers d'apports karstiques évaporitiques dans la Zone submédiane et carbonatés dans la zone de la chaîne du Rübli. Nous avons ainsi pu modéliser les écoulements en régime permanent en calant le modèle de transport des sulfates sur les résultats d'analyses chimiques réalisées à partir des nombreux prélèvements effectués le 6.4.1998 lors de la crue de printemps, celle-ci étant la plus importante pour la recharge de l'aquifère principal (Fig. 12). Nos connaissances sur les débits d'infiltration et d'exfiltration se résument alors aux estimations obtenues précédemment grâce aux calculs des bilans. Quant aux informations sur la to- 
Tab. 3. Bilans hydrauliques d'après l'étude GEOLEP (OEHE, 1999a).

\begin{tabular}{|c|c|c|c|c|c|c|c|c|}
\hline Profil & No & $\begin{array}{l}\text { Gradient } \\
\text { i [\%] }\end{array}$ & $\begin{array}{l}\text { Perméabilité } \\
\mathrm{K}\left[\mathbf{x 1 0}{ }^{-3} \mathrm{~m} / \mathrm{s}\right]\end{array}$ & $\begin{array}{l}\text { Section } \\
\text { saturée } \\
\mathbf{F}\left[\mathbf{m}^{2}\right]\end{array}$ & $\mathbf{Q}[\mathbf{l} / \mathbf{s}]$ & Q [Mio.m³/a] & $\begin{array}{l}\text { Différence } \\
{[\text { Mio.m³/a] }}\end{array}$ & Nature des échanges \\
\hline Gsteig Bode & 2 & 5 & 1.6 & 1000 & 80 & 2.5 & & \\
\hline Grund Süd & 3 & 6.6 & 1.3 & 1000 & 86 & 2.7 & 0.6 & $\begin{array}{l}\text { Infiltration de la rivière vers } \\
\text { la nappe et apports souterrains } \\
\text { par les karsts }\end{array}$ \\
\hline Grund Post & 4 & 2.8 & 3.7 & 1000 & 104 & 3.3 & 1.5 & $\begin{array}{l}\text { Infiltration de la rivière vers } \\
\text { la nappe et apports souterrains } \\
\text { par les karsts }\end{array}$ \\
\hline Hallenbad & 5 & 2.5 & 1 & 6000 & 150 & 4.7 & 11 & $\begin{array}{l}\text { Infiltration de la rivière vers } \\
\text { la nappe et apports souterrains } \\
\text { par les karsts }\end{array}$ \\
\hline Flugplatz & & & & & [Mio.m³/a] & {$[1 / \mathrm{s}]$} & & \\
\hline \multirow[t]{3}{*}{ Bilan global } & \multirow{3}{*}{\multicolumn{3}{|c|}{$\begin{array}{l}\text { infiltration }+ \text { apports par les karsts } \\
\text { infiltration } \\
\text { exfiltration }\end{array}$}} & & 4.4 & 138 & & \\
\hline & & & & & 1.2 & 38 & & \\
\hline & & & & & -6.4 & -204 & & \\
\hline
\end{tabular}

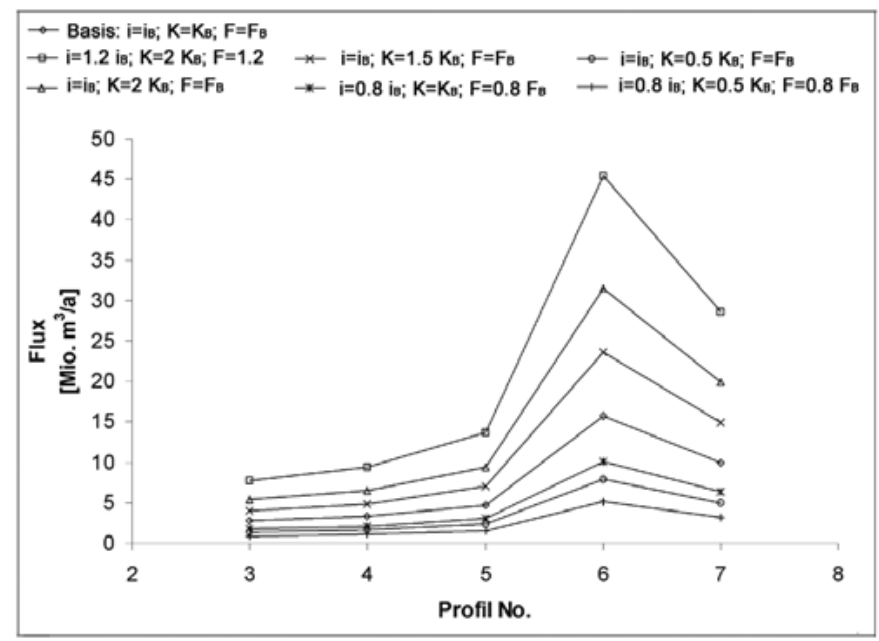

Fig. 11. Comparaison des différents scénarii de calcul des bilans d'après l'étude GEOLEP (OEHE, 1999a). Les paramètres i, K et F sont présentés dans le tableau 3 avec les valeurs utilisées pour le cas de base (Basis).

pographie et la structure de l'aquifère, elles proviennent d'une part des fichiers du Service Topographique Fédéral suisse (OFT, 1998), d'autre part de nos propres données acquises lors des campagnes de prospection géophysique (Fig. 13).

Si cette modélisation n'a pas réellement confirmé quantitativement les résultats obtenus par le calcul des bilans hydrau-
Tab. 4. Résultats de la modélisation (outils de calcul Budget Feflow) et comparaison de l'approche des bilans et de la modélisation.

\begin{tabular}{|c|c|c|}
\hline \multirow[b]{2}{*}{ Flux au travers des profils } & \multicolumn{2}{|c|}{ Résultats FEFLOW } \\
\hline & {$\left[\right.$ Mio $\left.\mathbf{~ m}^{3} / \mathbf{a}\right]$} & {$[\mathrm{l} / \mathrm{s}]$} \\
\hline IN & 7 & 230 \\
\hline \multirow[t]{2}{*}{ OUT } & 7 & 216 \\
\hline & 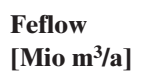 & $\begin{array}{l}\text { Bilans } \\
{\left[\text { Mio } \mathrm{m}^{3} / \mathbf{a}\right]}\end{array}$ \\
\hline Flux profil 4 & 1.6 & 3.3 \\
\hline Flux profil 5 & 3.8 & 4.7 \\
\hline Flux profil 6 & 5.5 & 15.8 \\
\hline \multirow[t]{2}{*}{ Flux profil 7} & 5.8 & 9.9 \\
\hline & $\begin{array}{l}\text { Feflow Bilans } \\
{[1 / s]}\end{array}$ & {$[\mathrm{l} / \mathrm{s}]$} \\
\hline Infiltration moyenne & 230 & 40 \\
\hline Exfiltration moyenne & 216 & 215 \\
\hline
\end{tabular}

liques (cf. tableau 4), elle a en revanche permis de simuler de manière tout à fait satisfaisante les apports en sulfates des versants triasiques de la Zone Submédiane à l'amont de notre modèle. Le panache de sulfates observé lors de notre campagne de mesures du 6.4.1998 a pu être reproduit de manière fidèle (Fig. 14). 


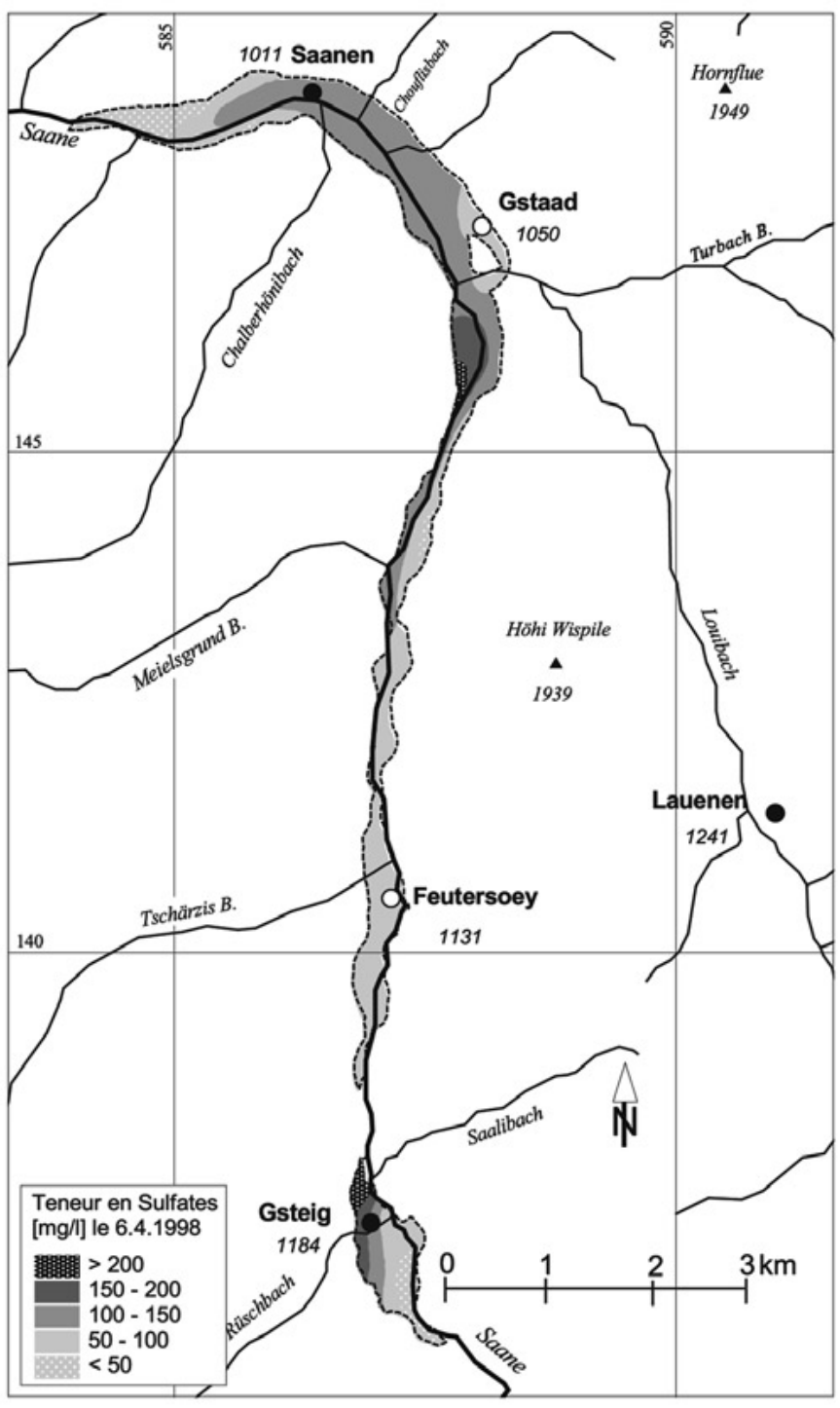

Fig. 12. Carte des teneurs en sulfates mesurées sur notre réseau d'observation des eaux souterraines le 6.4.1998.

\subsubsection{Synthèse}

Les résultats présentés ci-dessous ont été obtenus grâce aux calculs de bilans et à la modélisation effectués précédemment.

\subsubsection{Echanges rivière-nappe (alimentation indirecte de type A)}

\subsection{Infiltration par la rivière}

La Sarine joue un rôle important dans l'alimentation de la nappe (le débit d'infiltration moyen calculé sur une année est de l'ordre de 40 à 200 1/s, ce qui est sensiblement équivalent à l'alimentation directe par l'infiltration des précipitations).
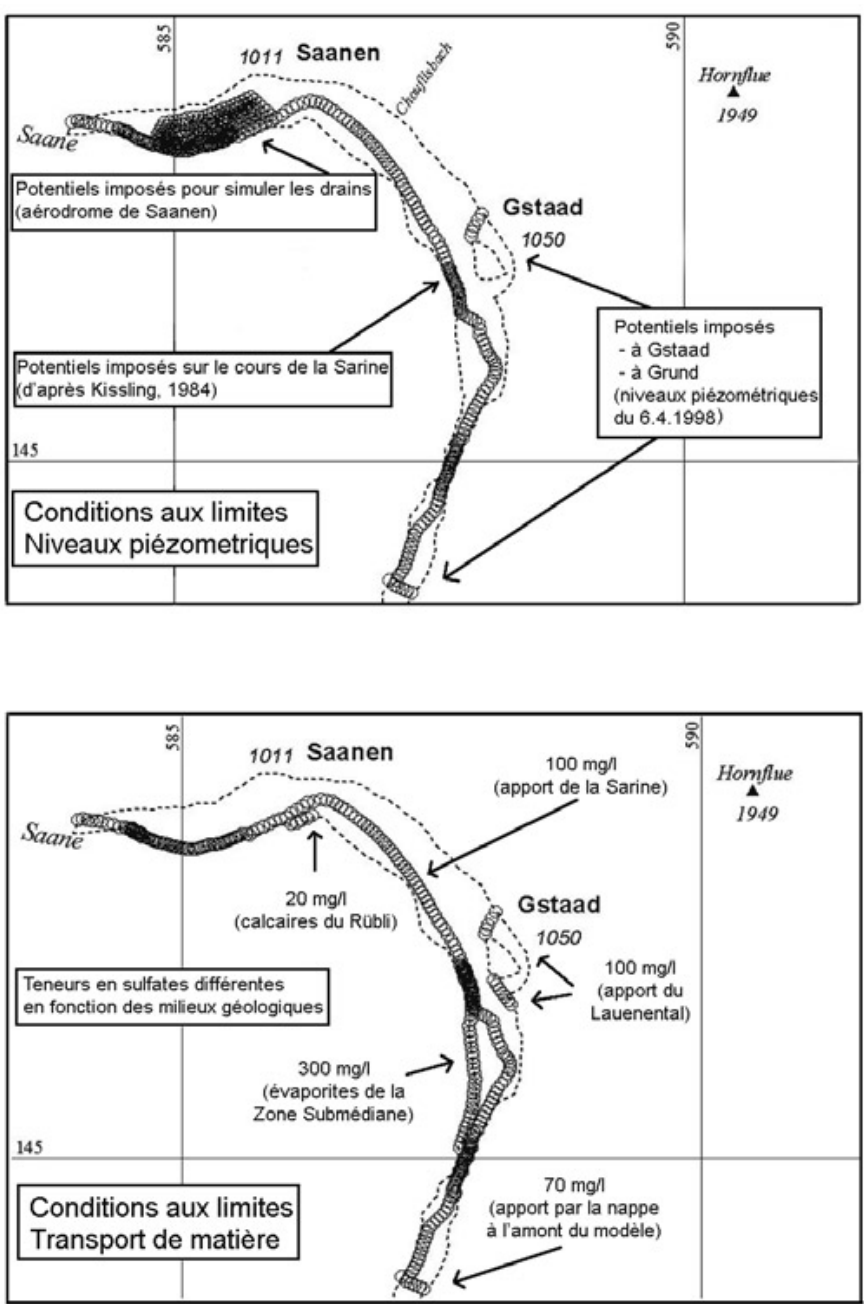

Fig. 13. Conditions aux limites piézometriques et pour le transport de matière utilisées pour la modélisation numérique des écoulements.

Cette alimentation indirecte s'effectue d'une part par l'infiltration en discontinuité hydraulique entre la rivière perchée et l'aquifère dans la région de Grund-Oey au sud de Gstaad (SB11, KB32 et KB33 où la Sarine est endiguée et légèrement surélevée par rapport aux terrains environnants), et d'autre part grâce à un mécanisme intermédiaire entre l'infiltration d'une rivière perchée et l'infiltration dans des zones de continuité hydraulique entre la Sarine et l'aquifère, du nord de Oey jusqu’à Rütti-Ebnit (SB5, SB6, FB13, FB14; Fig. 6).

\subsection{Exfiltration vers la rivière}

L'exfiltration de la nappe alluviale vers la Sarine a été étudiée qualitativement dans les zones situées à l'aval du glissement de terrain de Saali au nord de Gsteig (SB12; Fig. 6) ainsi qu'au nord du cône d'alluvions de Feutersoey et dans la zone de l'aérodrome de Saanen, soit une zone d'échange de 6 à $7 \mathrm{~km}$ de long, avec un débit moyen d'environ 200 1/s. 


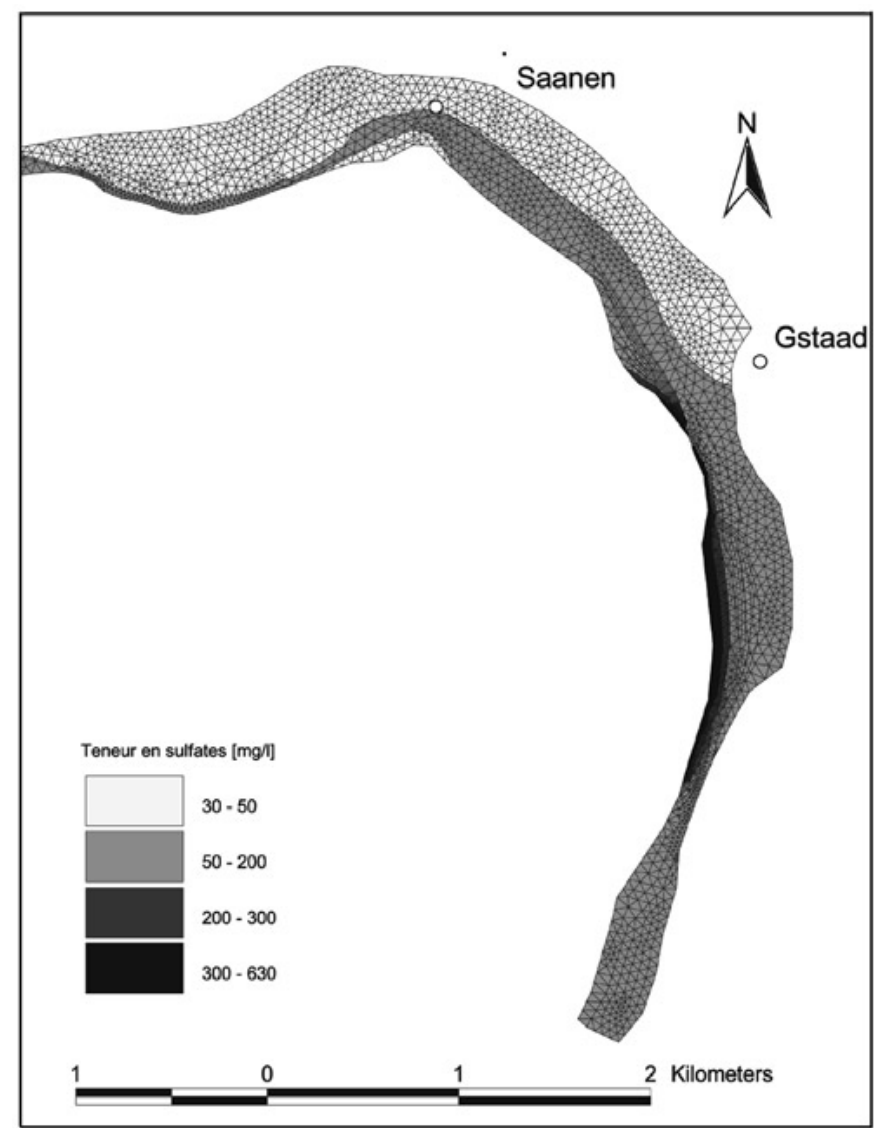

Fig. 14. Simulation des apports en sulfates; le panache calculé des teneurs élevées en sulfates est comparable à celui observé dans la réalité (cf. figure 13).

La construction de l'aérodrome et notamment de ses importants systèmes de drainage dans les années quarante a visiblement modifié les conditions naturelles d'échange entre la nappe alluviale et la rivière; en effet, l'exfiltration devrait y être importante en raison du seuil rocheux imperméable de Vanel qui ferme la vallée au nord-ouest. Cependant, les mesures de conductivité électrique de la nappe plus élevées en hiver qu'en été montrent une infiltration d'eau de la Sarine durant l'hiver dans le secteur amont de l'aérodrome due au système de drainage artificiel (Fig. 15). En revanche, les phénomènes d'exfiltration restent très nets tout au long de l'année dans le secteur aval: avant la crue de printemps, l'eau moins minéralisée de la nappe (FB1) exfiltre dans le secteur de Dorfrütti (A1), ce qui entraîne une baisse de la conductivité par rapport aux eaux situées en amont (A2). Après la crue, les eaux de la Sarine encore peu minéralisées sont enrichies dans ce même secteur par l'exfiltration de la nappe.

\subsection{Zones à régime intermédiaire}

Il s'agit de zones où les variations du niveau de la nappe permettent une inversion des relations hydrauliques entre celle-ci et la rivière, notamment dans la zone de Rütti-Ebnit où l'on

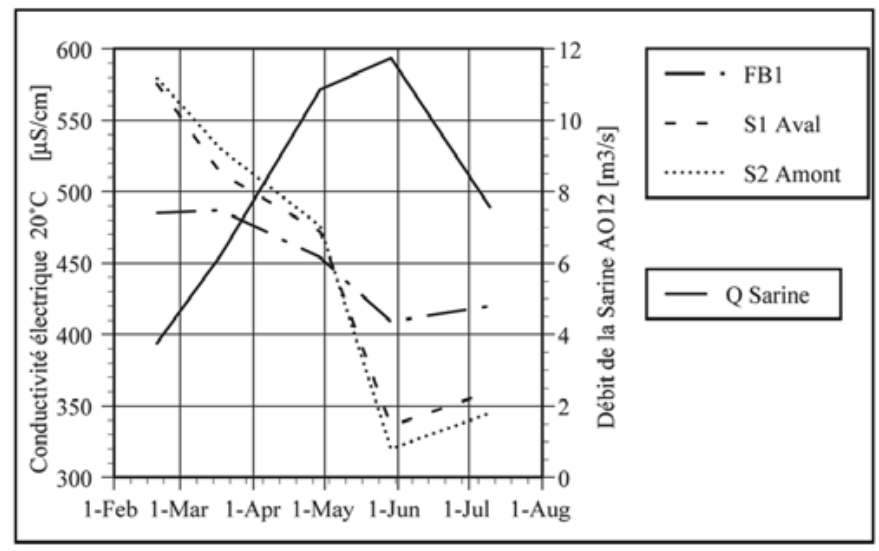

Fig. 15. Détail des échanges rivière-nappe à la hauteur de l'aérodrome de Saanen entre février et août 1998.

constate que le niveau de la nappe aux points FB13 et FB14 (Fig. 6) descend sous le niveau de la Sarine en période de basses eaux prononcées comme en novembre-décembre 1997. Un autre type de processus d'échange entre la Sarine et la nappe s'observe dans le secteur de Moosfang (SB5 et SB6; Fig. 6), où l'on observe un infiltration continue sur la rive gauche de la Sarine mais non sur sa rive droite, le niveau piézometrique au point SB6 se situant régulièrement au-dessus du niveau de la Sarine.

3.2.3.2. Alimentation par les karsts et les versants (alimentation indirecte de type B)

\subsection{Alimentation souterraine par les karsts}

L'ampleur de cette alimentation essentiellement souterraine n'étant pas connue de manière quantitative, nous l'avons évaluée à 50 à 100 1/s en nous basant sur notre connaissance des débits des sources karstiques des Préalpes (Mandia, 1991; OEHE, 1999a); elle joue donc un rôle non négligeable pour l'aquifère.

L'importante teneur en sulfates constatée dans la région de Gsteig Bode (SB12) au nord du village du même nom ainsi qu'au nord de Gstaad dans le secteur de Matte-Rütti (SB5 et SB6) est due aux exutoires karstiques des roches évaporitiques de la Zone des Cols ainsi que de la Zone Submédiane. Nous ne disposons malheureusement d'aucune donnée piézométrique permettant de déterminer la distribution des potentiels hydrauliques dans ces zones.

Il en va de même pour le karst calcaire de la chaîne du Rübli qui, d'après la carte des teneurs en sulfates de la nappe alluviale (Fig. 12), se vide vraisemblablement dans la plaine de la Haute-Sarine à la hauteur de Rübelsdorf, au sud du village de Saanen (FB2). Cela est confirmé par les basses conductivités électriques mesurées dans cette zone (Fig. 6) 


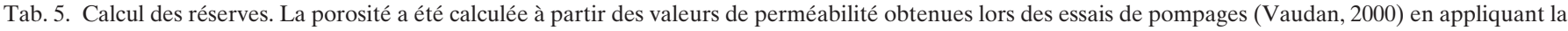
formule de Marotz d'après Hölting (1992): $\mathrm{P}[\%]=0.255+0.045 \ln \mathrm{k}_{\mathrm{f}}$. Les bassins sont représentés sur la figure 3.

\begin{tabular}{|c|c|c|c|c|c|c|}
\hline Bassin & $\begin{array}{l}\text { Surface } \\
{\left[\mathrm{m}^{2}\right]}\end{array}$ & $\begin{array}{l}\text { Porosité } \\
{[\%]}\end{array}$ & $\begin{array}{l}\text { Epaisseur moyenne } \\
\text { des sédiments }\end{array}$ & $\begin{array}{l}\text { Amplitude annuelle } \\
\text { des variations de la nappe } \\
\text { [m] }\end{array}$ & $\begin{array}{l}\text { Réserve } \\
\text { renouvelable } \\
{[\mathrm{m}]}\end{array}$ & $\begin{array}{l}\text { Réserve } \\
\text { permanente } \\
{\left[\mathbf{m}^{3} / \mathbf{a}\right]\left[\mathbf{m}^{3}\right]}\end{array}$ \\
\hline Gsteig & $666 ' 500$ & 14 & 20 & 1 & 93'000 & 1'900'000 \\
\hline Grund & $750 ' 670$ & 20 & 10 & 2 & $300 ’ 000$ & 1'500'000 \\
\hline Saanen & 3'418'140 & 21 & 20 & 1 & $720 ’ 000$ & $14^{\prime} 500 ’ 000$ \\
\hline
\end{tabular}

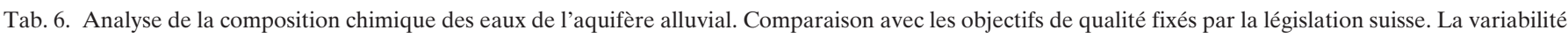
est à la fois spatiale et temporelle. Nous avons réalisé 55 mesures entre 1997 et 1998.

\begin{tabular}{|c|c|c|c|c|c|c|c|c|c|c|c|c|c|c|}
\hline \multicolumn{12}{|c|}{ CHIMIE } & \multicolumn{3}{|c|}{ BACTERIOLOGIE } \\
\hline Données & $\mathbf{N a}$ & $\mathbf{K}$ & Mg & $\mathbf{C a}$ & Cl & SO4 & NO3 & $\mathrm{HCO3}$ & Dureté & PO4 & NO2 & $\begin{array}{l}\text { Germes } \\
\text { aérobies }\end{array}$ & Entérocoques & E. coli \\
\hline [mg/l] & {$[\mathrm{mg} / \mathrm{l}]$} & {$[\mathrm{mg} / \mathrm{l}]$} & {$[\mathrm{mg} / \mathrm{l}]$} & {$[\mathrm{mg} / \mathrm{l}]$} & {$[\mathrm{mg} / \mathrm{l}]$} & {$[\mathrm{mg} / \mathrm{l}]$} & {$[\mathrm{mg} / \mathrm{l}]$} & ${ }^{\circ} \mathbf{f}$ & {$[\mathrm{mg} / \mathrm{l}]$} & {$[\mathrm{mg} / \mathrm{l}]$} & $/ \mathrm{ml} / 100 \mathrm{ml}$ & $/ 100 \mathrm{ml}$ & & \\
\hline Moyenne & 3.01 & 2.25 & 11.83 & 104.30 & 3.34 & 100.13 & 1.33 & 264.38 & 30.95 & 0.02 & 0.01 & 34 & 0 & 0 \\
\hline Max. & 11.5 & 8 & 17 & 149 & 15 & 210 & 11 & 365 & 46.25 & 0.06 & 0.03 & 58 & 0 & 0 \\
\hline Min. & 0.6 & 0 & 5 & 39 & 0.5 & 5 & 0.2 & 107 & 14.6 & 0.01 & 0 & 10 & 0 & 0 \\
\hline Oeaux & - & - & - & - & 40 & 40 & 25 & - & - & - & - & 100 & 0 & 0 \\
\hline
\end{tabular}

\subsection{Alimentation superficielle par les versants karstiques}

Il est intéressant de constater que la valeur moyenne de l'exfiltration de la nappe calculée ci-dessus correspond à un débit de $0.2 \mathrm{~m}^{3} / \mathrm{s}$. On peut considérer ceci comme la participation moyenne de la nappe alluviale au débit de base de la Sarine. Or, l'écoulement de base mesuré et retenu dans le cadre du calcul du $\mathrm{Q}_{347}$ est de 1.4 m³/s. La différence entre le débit d'exfiltration de la nappe vers la rivière et le débit de base correspond aux apports par le Reuschbach et le Saalibach qui drainent les aquifères karstiques de la Zone des Cols, ces deux torrents en étant apparemment les seuls exutoires de surface (Vaudan, 2000). Leur écoulement à charge élevée explique par ailleurs la forte teneur en sulfates de la Sarine en hiver.

\section{Synthèse des aspects quantitatifs et qualitatifs de la ressource}

\subsection{Aspects quantitatifs}

Le calcul des réserves a montré que les ressources en eaux souterraines de la Haute-Sarine sont importantes (cf. tableau 5): en effet, la réserve totale $R_{t}$ s'élève à plus de 19 mio $\mathrm{m}^{3}$ d'eau; cette valeur a été obtenue en faisant la somme de deux termes: d'une part la réserve permanente ou séculaire $R_{p}$ (Castany, 1967), c'est-à-dire la quantité d'eau contenue dans la partie de l'aquifère non-influencée par les variations saisonnières du niveau de la nappe (égale au produit du volume de sédiments aquifères saturés par la porosité des terrains considérés;

d'autre part la réserve renouvelable ou régulatrice $\mathrm{R}_{\mathrm{r}}$ (Cas- tany, 1967), correspondant au produit de la surface du bassin par la porosité des sédiments multiplié par l'amplitude annuelle des variations de la nappe.

\subsection{Aspects qualitatifs}

Comme le montre le tableau 6, les eaux de la nappe alluviale sont d'une qualité remarquable tant au niveau bactériologique qu'au niveau chimique, malgré une concentration en sulfates qui dépasse parfois les objectifs fixés par la législation suisse dans les zones d'alimentation par les karsts évaporitiques de la Zone de Cols et la Zone Submédiane. Ces teneurs élevées ainsi que la forte dureté de l'eau constituant plus une gêne technique qu'alimentaire (risques de corrosion ou de réaction avec le ciment), elles ne peuvent être considérées à elles-seules comme un facteur d'exclusion.

De plus, le fait d'être situées dans les zones amonts du bassin versant est généralement un gage de sécurité pour la qualité des eaux d'une nappe, les activités industrielles ou agricoles dangereuses y étant en principe très rares. Aussi les ressources en eaux souterraines de la Haute-Sarine peuvent être considérées comme une richesse très importante pour la vallée.

\section{Typologie des aquifères alluviaux de haute altitude}

Il nous a semblé important de comparer les caractéristiques propres à l'aquifère de la Haute-Sarine à celles d'autres vallées alpines. Plusieurs d'entre elles ont fait l'objet d'études scientifiques, mais peu de travaux ont essayé d'en faire la synthèse; c'est un des buts que s'est fixé la présente recherche. 


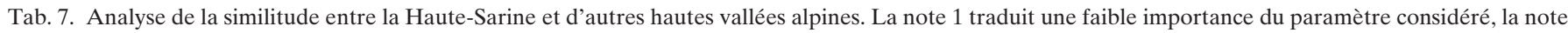
3 étant synonyme d'une très forte importance.

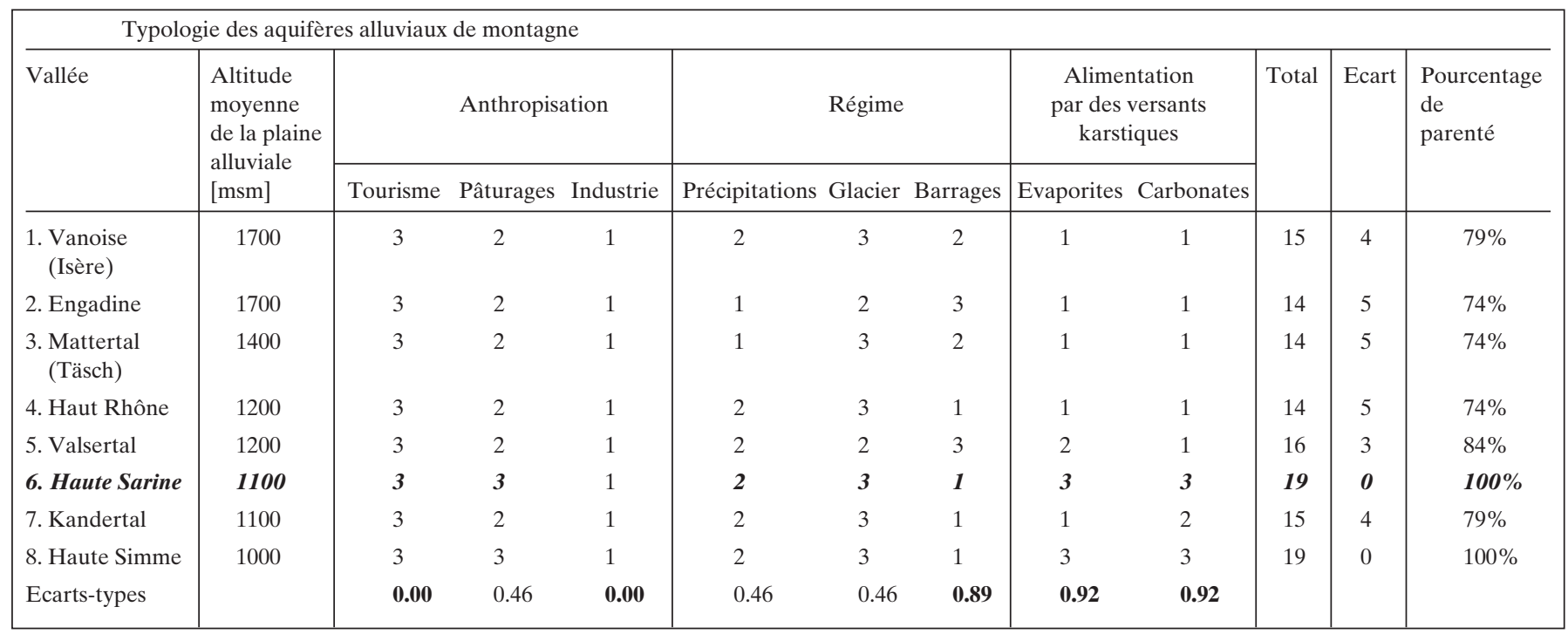

Afin de déterminer les caractéristiques spécifiques aux nappes alluviales de haute altitude, nous avons sélectionné plusieurs aquifères alpins (Fig. 16) pour en étudier les composantes pouvant influencer la gestion des ressources en eau. Des notes traduisant l'importance de chacun de ces facteurs ont ensuite été attribuées, la note 3 correspondant à une forte influence du facteur considéré sur la nappe. Après intégration de ces notes, on obtient une note globale qui nous a ensuite permis de calculer le pourcentage de parenté entre les aquifères considérés et l'aquifère de la Haute-Sarine (cf. tableau 7).

Nous avons ainsi pu isoler les caractéristiques communes aux différents aquifères étudiés ainsi que ce qui les différencie: dans tous les cas, on voit que les vallées étudiées ont une forte vocation pastorale et touristique, ce qui n'a rien de surprenant compte tenu du succès grandissant des sports de montagnes et $\mathrm{du}$ « tourisme vert » (cet aspect sera développé ultérieurement dans le chapitre sur le concept de gestion des eaux souterraines). Les activités industrielles y sont en revanche plutôt exceptionnelles.

Toutefois, on a pu constater que certains paramètres varient considérablement d'un aquifère à l'autre; il s'agit notamment de la nature des roches dans le bassin versant ainsi que, dans une moindre mesure, du nombre de barrages sur les rivières alimentant la nappe.

Comme mentionné dans le chapitre précédent, la présence d'évaporites ou de carbonates dans le bassin versant influence fortement la qualité de l'eau, pouvant limiter son exploitabilité: le contexte géologique est donc un facteur de différenciation très important. Quant aux barrages, ils agissent à plusieurs niveaux, notamment en limitant les fluctuations saisonnières de débit en aval, ce qui atténue l'effet des précipitations et des glaciers ainsi que les échanges rivière-nappe.
Grâce à ces principaux facteurs, nous pouvons dire que bien que chaque ombilic alpin ait ses particularités chimiques et hydrauliques qui justifient des investigations hydrogéologiques de base, il semble possible d'appliquer des modèles de gestion à long terme semblables aux eaux souterraines des aquifères alluviaux de haute altitude; il s'agit bien sûr de s'adapter aux conditions locales, mais les principes fondamentaux restent les mêmes.

\section{Concept de gestion des eaux souterraines en milieu alpin}

L'arrivée du tourisme dans les vallées alpines a nécessité la mise en place de réseaux de distribution d'eau potable ainsi que la multiplication de stations d'épuration des eaux usées (sources de pollutions ponctuelles), menaçant ainsi les ressources en eaux. Ainsi, parallèlement à la croissance de la consommation, on assiste à une augmentation de la pollution des nappes. La protection et l'utilisation rationnelle de ces ressources demandent un concept d'aménagement et de gestion à long terme des eaux souterraines. L'expérience du canton de Berne montre qu'il faut du temps et d'importants moyens financiers pour mener à bien un tel projet (dans le cas du canton de Berne, plus de 31 mio de francs suisses ont dû être investis sur trente ans); l'approche choisie est donc nettement plus « lourde » qu'une approche basée sur les caractères géomorphologiques.

\subsection{Première phase: investigations}

Cette étape est matériellement parlant la plus lourde, dans le sens où elle requiert l'utilisation d'importantes ressources scientifiques et techniques. Nous avons privilégié les méthodes 


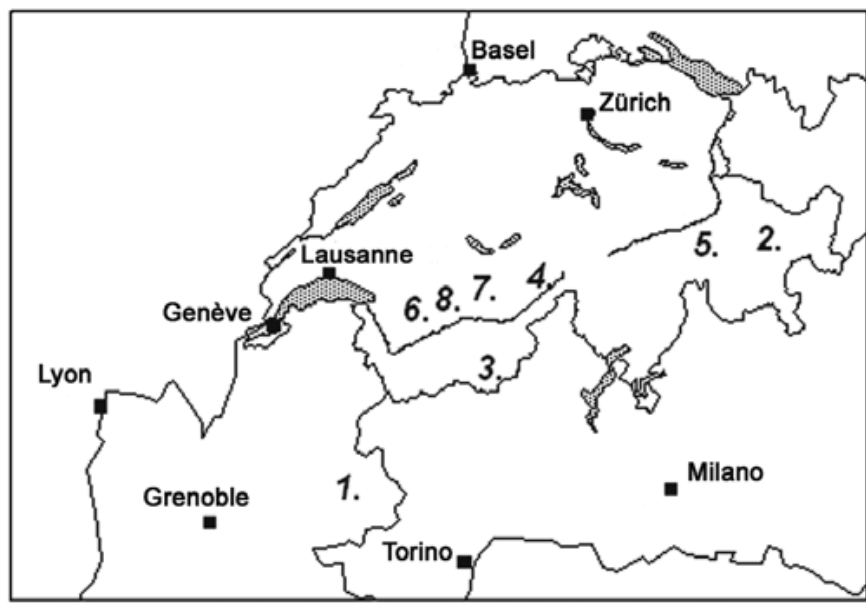

Fig. 16. Situation des vallées alluviales alpines considérées dans notre comparaison.

géophysiques (plus efficaces et plus rentables dans le cadre de la recherche d'eau) pour mettre en évidence les structures importantes des différents bassins aquifères de la plaine de la Haute-Sarine.

\subsection{Deuxième phase: concept de gestion}

L'application des principes de protection et d'utilisation des eaux souterraines (Blau et al., 1984) nous a permis d'établir une zonation de la nappe alluviale de la Haute-Sarine basée sur la qualité de l'eau et sur les quantités potentiellement exploitables (Fig. 17).

Au niveau de l'utilisation des eaux dans un but alimentaire, les zones « avec réserves » correspondent aux secteurs montrant les plus fortes concentrations en sulfates (comme les zones d'exutoires karstiques évaporitiques souterrains) ainsi qu'aux secteurs où la qualité des eaux est potentiellement menacée par des pollutions accidentelles et ponctuelles (aval des zones à bâtir et des zones industrielles). Au niveau quantitatif, l'aquifère de Zälg-Bode au sud de Feutersoey n'a pas été retenu comme très productif en raison de la présence du cône d'alluvions du torrent de Turbach, qui a complètement obstrué la vallée à l'aval de cet endroit, favorisant ainsi la mise en place de dépôts de type lacustres peu perméables.

L'utilisation de l'eau à caractère industriel concerne d'une part l'approvisionnement des entreprises nécessitant de l'eau pour leurs activités et d'autre part l'énergie (essentiellement par l'intermédiaire des pompes à chaleur dont l'utilisation est assez répandue en Suisse). Les principales restrictions sont essentiellement dues aux complications techniques qu'entraînent les fortes teneurs en sulfates et la dureté de l'eau. Nous avons également tenu compte de la productivité de la nappe, car certaines applications industrielles doivent pouvoir disposer d'une grande quantité d'eau.

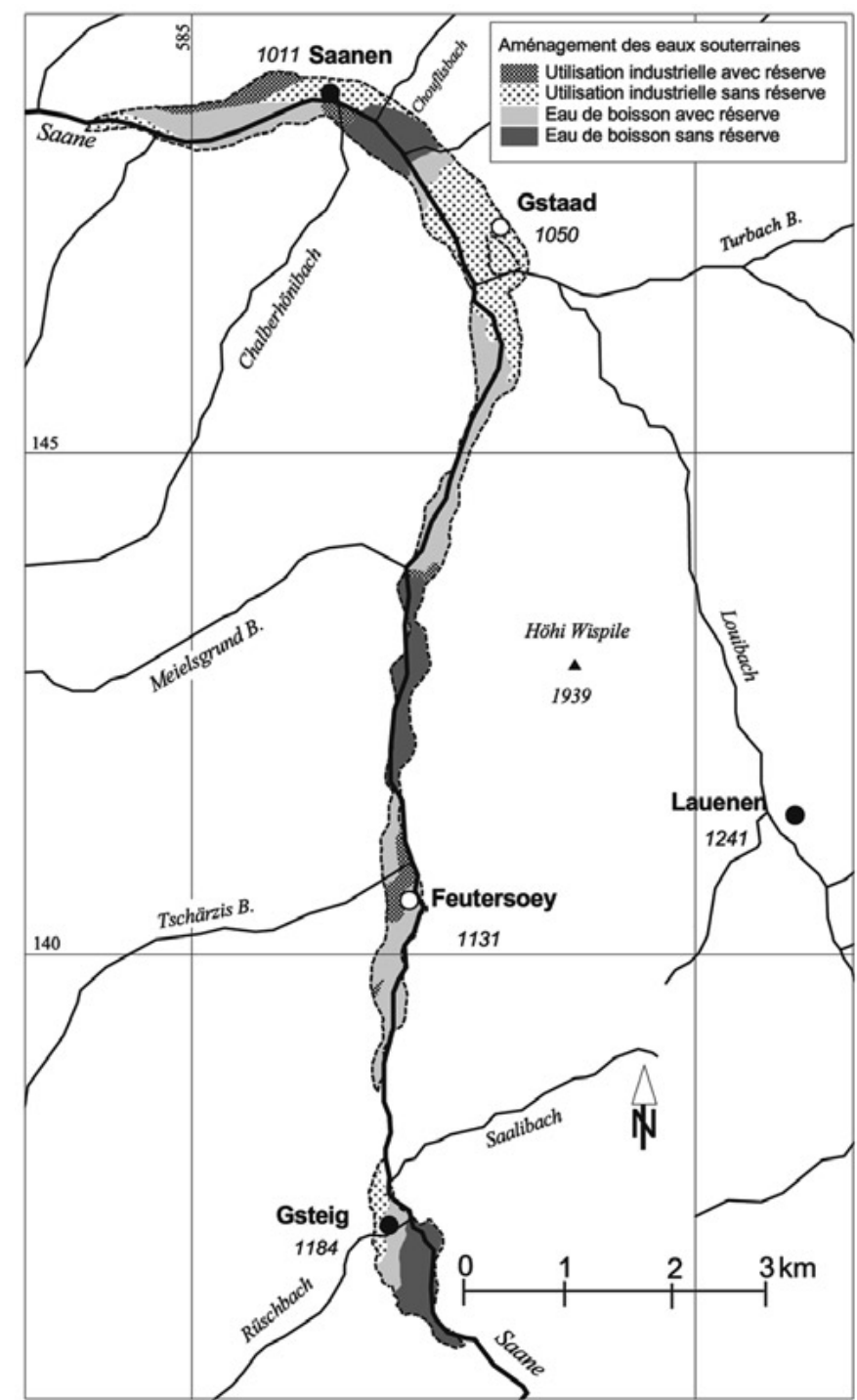

Fig. 17. Concept d'utilisation des eaux souterraines de la Haute-Sarine.

\subsection{Troisième phase: utilisation}

La gestion des eaux souterraines en milieu alpin peut se faire sur plusieurs bases. En principe, il s'agit d'utiliser en priorité le potentiel offert par les ressources renouvelables (Freeze, 1979).

Comme dans la plupart des vallées alpines, l'utilisation présente de la nappe alluviale n'est pas importante (cf. tableau 8). Les utilisations actuelles se font essentiellement sous la forme d'exploitation de chaleur au moyen de pompes à chaleur de type doublet; l'eau extraite dans ce contexte est donc entièrement restituée à l'aquifère. Aussi, seuls les $80^{\prime} 000 \mathrm{~m}^{3} /$ an pompés à la station de Chäppeli (qui ne sert plus que dans les périodes d'extrême affluence) sont véritablement retirés de la nappe. 
Tab. 8. Utilisation actuelle de la nappe alluviale de la Haute-Sarine. Il est important de relever que seule l'eau utilisée comme eau de boisson n'est pas restituée à l'aquifère, soit 80 '000 m³/a.

\begin{tabular}{|lll|}
\hline $\begin{array}{l}\text { Usage } \\
{[\mathbf{l} / \mathbf{m i n}]}\end{array}$ & $\begin{array}{l}\text { Concession OEHE } \\
{\left[\mathbf{m}^{\mathbf{3}} / \mathbf{a}\right]}\end{array}$ & Débit effectif \\
\hline Pompes à chaleur & $2{ }^{\prime} 330$ & $1200^{\prime} 000$ \\
Centre sportif & $6{ }^{\prime} 580$ & $300^{\prime} 000$ \\
Eau de boisson & $1 ' 500$ & $80^{\prime} 000$ \\
\hline Total 10'410 500'000 & & \\
\hline
\end{tabular}

Si nous comparons ces données avec le bilan des réserves calculé plus haut, nous constatons qu'à ce rythme, ces dernières ont encore de beaux jours devant elles. En effet, la consommation actuelle représente $7 \%$ des réserves renouvelables et $1.5 \%$ de l'infiltration directe des précipitations. Nous sommes bien en deçà des critères de la législation suisse.

\subsection{Mise en valeur à long terme}

Pour la vallée de la Haute-Sarine, les consommateurs potentiels sont les communes limitrophes ayant des problèmes d'alimentation en eau pendant les périodes d'affluence touristique. Les projets d'exploitation des ressources demandent une collaboration efficace entre ces communes ainsi que d'importantes études complémentaires sur les conséquences environnementales et les problèmes liés aux changements climatiques. En effet, si à moyen terme les glaciers et les importantes chutes de neige constituent la garantie d'une alimentation directe et indirecte importante, un réchauffement pourrait mener à une pénurie due soit à une qualité amoindrie, soit à une diminution importante des réserves, créant ainsi de nombreux conflits d'intérêts.

\section{Conclusion}

Cette étude a permis de mettre en évidence la complexité du fonctionnement de l'aquifère de la Haute-Sarine. Elle a notamment montré la grande importance de l'environnement géologique qui favorise l'alimentation de la nappe alluviale par le biais des réservoirs karstiques évaporitiques, mais qui peut également être un frein pour l'exploitation de la ressource en augmentant la dureté de l'eau par exemple. L'aquifère de la Haute-Sarine étant à plus d'un titre représentatif des ressources en eaux souterraines des hautes vallées alpines, la méthodologie adoptée peut ainsi être appliquée dans des cas similaires dans un but de protection et de gestion optimale de ces ressources.

\section{BIBLIOGRAPHIE}

Blau, R., Munchenberger, F., Trüeb, E. \& Würsten, M. 1984: Quantitative Erkundung von Lockergesteins-Grundwasserleitern am Beispiel Emmental. GWA: Gas, Wasser. Abwasser 63, 249-384.

Castany, G. 1967: Traité pratique des eaux souterraines. Dunod, Paris. 661pp.

DIERSCH, H.-J. 1998: FEFLOW: Finite Element subsurface Flow system: Reference manual, WASY, Institute for Water Resources Planning and Systems Research Ltd.

FLÜCK, W. 1973: Die Flysche der präalpinen Decken im Simmental und Saanenland. Beiträge zur geologischen Karte der Schweiz. Schweizerischen Geologischen Kommission, Bern. 87pp.

Freeze, R. A. \& Cherry, J. A. 1979: Groundwater. Prentice-Hall Inc., New Jersey. 461pp.

Fürst, J., Nachtnebel, H. P. \& Reichel, G. 1990: Multi-cell model for modelling and managing aquifers in narrow alpine valleys. Water resources in mountainous regions. AIH, Ed., Lausanne.

Hölting, B. 1992: Einführung in die Allgemeine und Angewandte Hydrogeologie 4. Auflage. Enke Verlag, Stuttgart, 79pp.

JuIF, L. 1991: Hydrogéologie de la haute-montagne: approche du fonctionnement hydrodynamique des aquifères de Vanoise. Thèse Université de Franche-Comté, 4-64, 158pp.

KissLing, Z.I.A. 1984: Projekt II für die Verbauung der Saane, Kanton Bern, Oberingenieur Kreis I, Bern.

MAISCH, M. 1982: Zur Gletscher und Klimageschichte des alpinen Späglazials. Geographica Helvetica 2, 93-104.

MANDIA, Y. 1991: Typologie des aquifères évaporitiques du Trias dans le bassin lémanique du Rhône (Alpes occidentales). Thèse EPFL No 948.

Menzel, L., LAnG, H. \& Rohman, M. 1989: Table 2.1: Evaporation réelle annuelle moyenne 1973-1992. Atlas Hydrologique de la Suisse.

Nicoud, G. \& Maillet-Guy, G. 1990: L’hydrogéologie des vallées glaciaires alpines à partir de l'exemple du bassin chambérien. Water resources in montainous regions. IAH, Ed., Lausanne.

OEHE 1983: Hydrogeologie Emmental Teil IV. Office de l'Economie Hydraulique et Energetique du Canton de Berne, Berne, D32-D39.

OEHE 1989: Seeland: Infiltration aus Hagneckkanal und Alter Aare. Office de l'Economie Hydraulique et Energetique du Canton de Berne, Berne, $52-53$.

OEHE 1994b: Hydrogeologie Haslital. Office de l'Economie Hydraulique et Energetique du Canton de Berne, Berne, 126-133.

OEHE 1999: Nutzungs-, Schutz- und Ueberwachungskonzept für den Grundwasserleiter des Seelands: Synthesebericht. Office de l'Economie Hydraulique et Energetique du Canton de Berne, Berne.

OEHE 1999: Hydrogeologie Oberes Saanetal. Office de l'Economie Hydraulique et Energetique du Canton de Berne, Berne.

OEHE 1999: Hydrogeologie Oberes Simmental. Office de l'Economie Hydraulique et Energetique du Canton de Berne, Berne, 23-29.

OfT, O.F.D.T. 1998: DHM25 Matrixmodelle Karten Nr 1245. 1246 und 1266, Auftrag Nr. 3310-98037.

Primault, B. 1972: Etude meso climatique du canton de Vaud 1:200 000 \& 1: 500 000. Office Cantonal Vaudois de l'Urbanisme, Lausanne.

VAUDAN, J. 2000: Fonctionnement et gestion des aquifères alluviaux de haute altitude, cas de la Haute-Sarine (Alpes suisses). Thèse EPFL n²244, Lausanne.

Manuscrit reçu le 4 février 2003

Révision acceptée le 30 mars 2005 
